\title{
Contenido y audiencia de los informativos: Canal Sur TV y TVE en Andalucía
}

Una aproximación metodológica

\author{
Dr. Carlos A. Guerrero Serón \\ Departamento de Comunicación. Universidad de Sevilla.
}

\begin{abstract}
En este artículo sintetizo los resultados de una investigación que, financiada por el Grupo de Investigación «Desarrollo y Cambio Socioeconómico», tuvo como objetivo conocer la influencia política en los contenidos de los informativos. Para realizar el contraste se analizaron los informativos de Canal Sur y las desconexiones informativas de TVE-Andalucía en la consideración de que, en estos momentos, se da una situación idónea para poner a prueba la hipótesis habida cuenta que conviven dos televisiones públicas, la andaluza, controlada por el Partido Socialista, y la nacional, bajo el control del Partido Popular. Los resultados del análisis de contenido se cotejaron con los perfiles respectivos de sus audiencias y con los generales de la franja horaria, para comprobar si la audiencia específica se aparta sensiblemente de la tipología general del periodo horario y, en su caso, si la selección de los valores/noticia por parte de los emisores -periodistas- están en disonancia con lo que el público espera.
\end{abstract}

\section{Marco teórico}

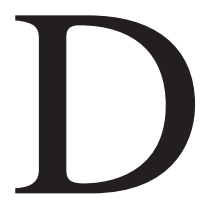

esde principios de siglo, los investigadores de la comunicación centraron sus preocupaciones en la influencia de los medios sobre los públicos (McQuail y Windahl, 1984:34-35). Alentados por los paradigmas evolucionistas dominantes, provenientes de pensadores como Comte, Spencer, Tönnies o Durkhein (DeFleur y Ball-Rokeach, 1986:215) las primeras investigaciones sobre los medios de comunicación admitieron como buenas las explicaciones que postulaban los efectos directos de los mensajes sobre los individuos: los medios poseían la capacidad de moldear a un público que percibía y reaccionaba a los mensajes de forma más o menos uniforme. Se daba por hecho la relación directa entre el estímulo y la respuesta obtenida. 
Entre los años 20 y los 40 se producen una innumerable cantidad de investigaciones sin un corpus epistemológico delimitado, pero van acumulando datos y vislumbran nuevas perspectivas que, durante la posguerra, comienzan a cristalizar en modelos y teorías cada vez más sólidos (Roda, 1989:73) ${ }^{1}$ siempre dentro de esquemas mecanicistas. La "validez de la prueba" empírica frente a los procedimientos hermenéuticos, continúa siendo una exigencia común, sin duda, por la necesidad de obtener respuestas inmediatas a los problemas planteados.

Este entorno científico es buen caldo de cultivo para que florezcan teorías que, como el behaviorismo de J.B. Watson, se ven apoyadas por la publicación de algunos estudios sobre los resultados de determinadas campañas comunicativas -Vgr.: La invasión desde Marte de Cantril, Gaudet y Herzog (1940)²- que "demuestran" la validez de los planteamientos seguidos. También en este tiempo se producen algunos acontecimientos que van a tener importancia en los paradigmas y metodologías posteriores, como la publicación del Curso de Lingüística general de Saussure que supone una ruptura epistemológica con los estudios lingüísticos que hasta entonces habían imperado al preconizar lo que más tarde se conocería como estructuralismo lingüístico (Rodrigo, 1989:55).

Lógicamente, el asentamiento de las diversas escuelas trae consigo debates entre lo cualitativo y lo cuantitativo, las metodologías experimentales frente a las heurísticas, etc. consecuencia, por otra parte, de la situación que se vivía en los estadios más teóricos o filosóficos de la ciencia después de la inflexión operada en el saber científico tras la reunión de Viena. En estos años se impone el empirismo sociológico en el ámbito universitario con una profusa utilización de sondeos como instrumentos de previsión de acontecimientos sociales. La Escuela de Chicago, que representaba el funcionalismo de Talcott Parsons, entra en lucha por la hegemonía universitaria con la Universidad de Columbia, representada por el empirismo de Lazarsfeld ${ }^{3}$, hasta que la mediación de Merton consigue aproximar a ambos estableciendo como modelo dominante las El estudio de Decatur de Lazarsfeld, Berelson y

\footnotetext{
RODA (1989): Los medios de comunicación de masas. Su influencia en la sociedad y en la cultura contemporáneas. Madrid. C.I.S. p. 73.

2 Como dice Roda "En conjunto, el periodo coincide con el proceso de construcción de conceptos y desarrollo de métodos que se utilizarán habitualmente en la estimación empírica del impacto de la comunicación”.

3 RODRIGO ALSINA, M. (1989): Los modelos de la Comunicación. Madrid. Tecnos. pp. 45-46.
} 
Gaudet ${ }^{4}$ constituyó, probablemente, el primer hito en el cambio de criterios respecto a los efectos directos de los mass media ${ }^{5}$. En esta investigación se descubre el papel que desempeña el grupo primario y los líderes de opinión en la formación de actitudes y la toma de decisiones de los individuos. Esto provoca un cambio de opinión respecto a la eficacia de los medios cuya consecuencia es un movimiento pendular hacia el polo opuesto: los medios son ineficaces para la formación de actitudes si no cuentan con otras circunstancias que apoyen sus informaciones. Se plantea entonces la teoría de la comunicación a dos niveles (two step flow) tras la publicación en 1944 de los resultados de la investigación en el libro "La influencia personal"6 por parte de Katz y Lazarsfeld. Más tarde, Klapper (1974) ${ }^{7}$ se encargó de sistematizar los conocimientos que abundan en las hipótesis de los efectos mínimos y Fagen $(1967)^{8}$ continua esta línea aplicándolos a la comunicación política.

Con este bagaje de conocimientos y técnicas de las que Lazarsfeld con su capacidad de integrar los modelos matemáticos con los planteamientos hermenéuticos- fue indudable pionero, pudieron plantearse nuevos avances en el análisis de los componentes del sistema de comunicación. El mismo Lazarsfeld junto a Berelson comienzan a estudiar otros aspectos de los mensajes centrando su interés en características paralingüísticas de los mismos (posición, apoyo icónico, caracteres tipográficos,...) introduciendo los criterios modernos de esta técnica.

Otros autores como Carl I. Hovland, con la meticulosidad y hermetismo que le caracterizaron, comienzan a publicar resultados de investigaciones que ponen en evidencia efectos a largo plazo de determinados tipos de comunicaciones que permiten establecer algunas hipótesis sobre el hecho de que, en ciertos casos, el paso del tiempo realza los efectos del mensaje que había quedado 'latente' durante un tiempo (Rodrigo, op. cit: 68).

\footnotetext{
LAZARSFELD, P. F.; BERELSON, B. Y GAUDET, H. (1944): The people's choice. NuevaYork, Duell, Sloan and Pearce. Citado por Roda Fernández, op. cit. pag. 74.

5 Hablar de los "padres fundadores" de la disciplina implica referirse indiscutiblemente a la Escuela de Chicago y aquí resulta imprescindible reseñar la labor pionera de Thomas y Znaniecki, quienes en 1920 publican los resultados de su trabajo empírico sobre la integración de los campesinos polacos, donde se analiza el significado funcional de la prensa en la organización social (Principio de Thomas) y que para Bardin (1986:10) constituye el precedente remoto del moderno del análisis de contenido.

6 KATZ, E. y LAZARSFELD, F. (1979): La influencia personal: el individuo en el proceso de comunicación de masas. Barcelona. Hispano Europea.

7 KLAPPER, J.T. (1974): Los efectos de las comunicaciones de masas. Aguilar. Madrid. (Edición original inglesa en 1960. Free Press. New York)

8 FAGEN, Richard R. (1967): Política y Comunicación. Buenos Aires. Paidós. (Edición inglesa, 1966, Little, Brown and Company. Boston)
} 
202 Contenido y audiencia de los informativos: Canal Sur TV y TVE en Andalucía

Desde la corriente empírico experimental o de "la persuasión", de raíz psicológica, se estudiaron factores relativos a la audiencia (interés del contenido para el público, selectividad en la exposición, en la percepción y en la memorización en función del contenido y de otras variables, como el nivel de instrucción, la edad, el sexo, etc.) y factores vinculados al mensaje (credibilidad de la fuente, el orden de argumentación, la exhaustividad de las argumentaciones).

Los estudios sobre el consumo de los media comienzan a incluir análisis del contenido de los programas, de las características diferenciales de las audiencias y de los motivos o gratificaciones que reciben los oyentes con la exposición a determinado contenido en lugar de otra alternativa. Este tipo de análisis requiere un diseño donde se interrelacione 1) la finalidad práctica (por qué la gente se expone a determinados programas), 2) su importancia teórica (conceptualización de problemas), y 3) una metodología capaz de obtener la información que se precisa (Wolf, op. cit.: 52-54). Es decir, estamos en presencia de investigaciones cuya metodología requiere la utilización de técnicas cuantitativas y cualitativas y que permiten establecer orientaciones que se van acercando cada vez más al estudio de los procesos y los fenómenos comunicativos relacionados; es decir, estos estudios abren las perspectivas de análisis desde los medios hacia el contexto social en el que operan.

La corriente de los usos y gratificaciones que surge algo más tarde de la mano del funcionalismo, parte del supuesto de que los públicos utilizan los medios en relación con sus propias necesidades, por lo que obtiene gratificaciones, y no siempre se ajustan a los fines pretendidos por los comunicadores o emisores. En palabras de Merton (1982) “... los mensajes son disfrutados, interpretados y adaptados al contexto subjetivo de experiencias, conocimientos, motivaciones". El receptor pasa a tener una consideración activa, capaz de influir en el emisor -ya sea a través de los estudios de comportamiento que informan a los emisores sobre los gustos y preferencias de sus públicos, ya mediante vía directa (cartas de lectores), a través de organizaciones, etc.- que obligan a los comunicadores a tenerlos en consideración. En otras palabras, el receptor deja de ser un objeto pasivo para el emisor (Wolf: 79). Las gratificaciones de los destinatarios pueden estar relacionadas con el contenido específico del mensaje o con la exposición al medio en sí misma, con lo que el estudio del contenido puede tener un carácter secundario en el estudio de las reacciones de la audiencia (McQuail, 1975:55). 
Los modelos que se construyen a partir de entonces (v.gr.: Westley y MacLean, Maletzke, ...) comienzan a tener en cuenta estas consideraciones dentro y como parte importante del proceso de comunicación (Windahl, Signitzer\&Olson, 1992:120-132).

A partir de los años setenta cuando aún se mantiene viva la polémica ideológica entre la communications research y la escuela crítica, empiezan a sentirse cambios de orientación en el estudio de los medios tendentes al análisis de sus efectos a largo plazo que, como valor añadido, van a propiciar un acercamiento entre los paradigmas tradicionalmente enfrentados. Las nuevas tendencias van a centrar su interés en el estudio de la cobertura global de los medios por áreas temáticas, mediante metodologías integradas y complejas que van a tratar de reconstruir el proceso con el que el individuo modifica su propia representación de la realidad social. Frente a las metodologías anteriores, centradas en el estudio de casos mediante encuestas que analizan los cambios de actitud, las nuevas metodologías cambian su orientación desde los efectos sobre actitudes y valores hacia los efectos sobre el sistema de conocimiento -efecto cognoscitivo- que los individuos se estructuran a partir de lo que asimilan de las comunicaciones de masas, que son efectos acumulativos, y no puntuales, dentro de un proceso que tiene una dinámica interna y, a la vez, está relacionado con otros procesos previos o simultáneos.

En estos últimos años, se ha vuelto a considerar el alto poder de los medios aunque basado en consideraciones más complejas de las propugnadas por las teorías iniciales. Los enfoques marxistas sobre el contenido de los medios resaltan el importante papel que desempeñan en la legitimación del capitalismo y las sociedades burocráticas; se han detectado nuevos tipos de efectos, como el cultivo, la agenda-setting o la espiral de silencio que orientan hacia una nueva categoría de efectos no unidireccionales. En esta última fase investigadora sobre los efectos se realza el "poder", sintetizando el contenido de los medios con los factores individuales, frente a la "ineficacia" anterior a los setenta y a la "omnipotencia" que se les atribuía inicialmente (Windahl, Signitzer\&Olson, 1992:179).

Los más recientes investigadores enfatizan una serie de factores interrelacionados de tipo cultural (Bordieu), de dependencia personal del medio (DeFleur y Ball-Rockeach), motivaciones individuales temporales (Hornik), percepción de la realidad (Nordersteng), etc. Quizás la más reciente sea la de Rosengren que sugiere otros dos factores: los recursos indivi- 
204 Contenido y audiencia de los informativos: Canal Sur TV y TVE en Andalucía

duales y el control ejercido por los agentes de socialización (familia, organizaciones, ...). Por último, en referencia a las posibilidades y cambios que se prevén con la introducción de las nuevas tecnologías, hago mías las palabras de McQuail (1997:147)9.

"La vieja idea de que los medios crean su propia audiencia está lejos de extinguirse a pesar de la gran autonomía e influencia de los receptores. Esto no es el resultado de una experta manipulación de la inteligencia del marketing y la publicidad. Hay todavía intérpretes, interpretaciones, reportajes, noticias que se muestran día a día por la prensa, la radio, el cine, video o televisión que unen a un grupo de espectadores en algo que les gusta mucho más que su experiencia cognitiva o imaginativa" 10 .

Es con relación a estas últimas teorías y modelos respecto a las que se enfoca esta investigación. En otras palabras, el propósito es establecer una aproximación entre el contenido de los medios y las características de la audiencia, siguiendo los criterios desarrollados por las teorías de los efectos a "largo plazo". Por supuesto, la tarea es demasiado ingente para ser abordada en una investigación de las características planteadas, requeriría un enfoque mucho más globalizador. Pero, al final, una investigación está delimitada por dos variables instrumentales que, a la postre, inciden decisivamente en los planteamientos metodológicos: el tiempo y los recursos económicos. Ambos son escasos en nuestra situación, por lo que se ha procurado obtener la mayor productividad de los recursos disponibles.

\section{Objetivos de análisis}

Los objetivos que nos planteamos en esta investigación son modestos. Dentro del enunciado general de establecer una aproximación entre el contenido de los medios y las características de la audiencia, subyacen otros objetivos parciales o subordinados. El primer objetivo instrumental es conseguir poner a punto una herramienta de análisis que sustituya a los tradicionales "vaciados de prensa" en forma de selección de informaciones referidas a determinadas personas o instituciones. En este sentido, la técnica que se utiliza se diseñó inicialmente en 1994 para el Instituto de Marketing y Comunicación, S.L. (IMC) bajo el nombre comercial de Sistema de Control

\footnotetext{
9 MCQUAIL, D. (1997): Audience Analysis. London. Sage.

${ }^{10}$ Las traducciones de las dos últimas citas son mías.
} 
de Información y Publicidad (C.I.P.) con el propósito de desarrollar en la Comunidad Autónoma Andaluza un sistema de medición de la comunicación objetivo y sistemático, que pudiera responder a criterios científicos mediante la utilización de una metodología contrastable. Para ello, se siguieron criterios metodológicos de las escuelas más empiristas en el análisis de contenido textual" con aportaciones de criterios "ad hoc" para poder disponer de valoraciones cuantitativas y cualitativas. En un primer momento, el Sistema CIP se aplicó a la prensa escrita exclusivamente, y es a partir de noviembre de 1997 cuando se comienza a hacer también de los informativos de televisión, adaptando algunas variables a las características peculiares del medio pero manteniendo los criterios metodológicos básicos.

Entre los objetivos de conocimiento se planteó la hipótesis de la influencia política -de los políticos- en los contenidos de una manera más o menos larvada o sinuosa. Consideramos que la situación andaluza de estos momentos es idónea para realizar tal contraste habida cuenta que conviven dos televisiones públicas, la andaluza, controlada por el Partido Socialista, y la nacional, bajo el control del Partido Popular ${ }^{12}$ (Esto también sucede en la prensa y la radio, pero de una forma más sutil al estar mediadas por el poder económico).

Utilizaremos como unidad de investigación los informativos diarios, como mejores exponentes de la intencionalidad ideológica. Correlativamente, compararemos los perfiles de las audiencias de los informativos con los generales de la franja horaria para ver si el adoctrinamiento cae en terreno abonado y, complementariamente, trataremos de comprobar si la audiencia específica se aparta sensiblemente de la tipología general del periodo horario y, en caso de confirmarse la hipótesis, trataremos de ver si la tendencia es a que el perfil se adapte al contenido general -o línea general-, es decir, los contenidos más "románticos" a los grupos más "sensibles", los más agresivos a los más reivindicativos, etc. o, por el contrario, si se evidencia de que la audiencia de los informativos es similar a la del conjunto del periodo -coloquialmente, si el público ve "lo que hay"- habrá que pensar que la

\footnotetext{
${ }^{11}$ NAVARRO, Pablo-DÍAZ Capitolina (1994): “Análisis de Contenido” en DELGADO, J.M. y GUTIERREZ, J.coordinadores- Métodos y técnicas cualitativas de investigación en ciencias sociales. Madrid. Síntesis Psicología. Pps.177-224.

- RUIZ OLABUÉNAGA, J.I./ISPIZUA, Ma Antonia (1989): La descodificación de la vida cotidiana. Bilbao. Universidad de Deusto. Capítulo VII. pp.181-218.

12 A esta variable le suele suceder como a la negociación "de pasillos y reservados" en los manuales sobre técnicas de negociación, que nadie las menciona pero es la que realmente se utiliza (!).
} 
206 Contenido y audiencia de los informativos: Canal Sur TV y TVE en Andalucía

selección de los valores/noticia por parte de los emisores -periodistas- están en disonancia con lo que el público espera. En otras palabras, los periodistas tendrían un grave desconocimiento sobre las necesidades y gustos de sus audiencias, aunque

“...la referencia y la alusión a las necesidades, a las exigencias de los destinatarios es constante, y en las propias rutinas productivas están encarnadas convicciones implícitas sobre el público, solo que no están muy ajustadas a la realidad. Los periodistas explican su conocimiento sobre el público haciendo referencia a su profesionalidad, esfuerzo y experiencia, pero está demostrado que estar inmersos en el absorbente mundo de las noticias no aporta criterios ciertos sobre los gustos del público. Por otra parte, se da la circunstancia de que la fuerte estructura organizativa de los emisores en los medios supone que a menudo los comunicadores no están implicados en el comunicar, y cuando lo están, no están necesariamente comunicando en primer lugar con el público en sí, sino con públicos muy específicos que pueden estar constituidos por los colegas, potenciales anunciantes o miembros de otras instituciones" (ALDANA, 1998:77-78)13

\section{Metodología. Criterios científicos}

Las reglas de análisis preconizadas por Berelson fueron puestas en cuestión y ampliadas posteriormente, pero iniciaron un camino que "...indican la inquietud de este periodo por trabajar con muestras reunidas de forma sistemática, por interrogarse sobre la validez de los procedimientos y de los resultados, por verificar la fidelidad de los codificadores e incluso por medir la productividad del análisis" (Bardín, op. Cit.: 14).

Los años siguientes, cuando muchos investigadores, incluido el propio Berelson, parecen decepcionados por el escaso avance de las técnicas de análisis, se produce un reflotamiento del interés en esta técnica como consecuencia, según Krippendorf (1990:20), de la incorporación de eminentes científicos sociales, la definición de nuevos conceptos (estereotipo, actitud, símbolo,...), el desarrollo de la estadística y la inclusión de sus resultados en

\footnotetext{
13 ALDANA, M. (1978): La información en TV: papel de los informativos en la lucha por la audiencia en A.E.D.E.M.O. $83^{\circ}$ Seminario. $14^{\circ}$ Seminario sobre audiencia de televisión. Granada. Febrero, 1998.
} 
trabajos de mayor envergadura, lo que propició la adopción de nuevas vías metodológicas provenientes de campos muy diversos que confluyen en el interés por el análisis de contenido.

La disputa entre las metodologías o puntos de vista cuantitativos y cualitativos se recrudece, los primeros con su centralidad en las frecuencias de observación y, los segundos, en las presencias o ausencias de características. En esta disputa adquiere gran importancia la labor de Osgood quien introduce y perfecciona nuevas técnicas de análisis (de aserciones estimativas y de contingencia, sobre todo) que permiten, por un lado, flexibilizar los criterios anteriores de objetividad, entendidos en un sentido de exhaustividad o minuciosidad y, por otro, tomar conciencia de su función de inferencia (Bardín, op. cit.: 16).

La generalización del ordenador viene a dar otro impulso al análisis de contenido, junto al creciente interés por el análisis de componentes no verbales y las aportaciones de los estudios lingüísticos. El ordenador facilita la labor de clasificación y frecuencia pero exige una mayor dedicación a las labores de codificación de registros y posibilita, o facilita, la utilización de pruebas estadísticas con un alto número de variables, a la vez que permite la utilización de técnicas imposibles de utilizar "a mano", como los análisis factoriales. Todo ello colabora a ampliar el campo de análisis a disciplinas cada vez más diversificadas permitiendo la utilización de categorías estandarizadas. Ahora el cumplimiento de los requisitos del método científico en el diseño de la investigación autorizan a utilizar las reglas de validez y fiabilidad exigibles a las variables (homogeneidad, exhaustividad, objetividad, exclusividad) lo que faculta al analista para deducir contenidos de los mensajes de naturaleza psicológica, sociológica, etc., es decir, existe la posibilidad de establecer tratamientos de causalidad que facultan la inferencia en condiciones científicas.

Para construir el aparato metodológico, y en el posterior tratamiento y análisis de los datos, hemos seguido los criterios emanados de la bibliografía citada, particularmente de los autores Bardin, Krippendorf, Navarro y Díaz y Olabuénaga e Ispizua.

En lo que respecta a los datos sobre audiencias de los telediarios, se han utilizado los ficheros del estudio de metodología tracking "Medios y Audiencias de Andalucía" del Instituto de Marketing y Comunicación, S.L. (IMC) referidas al tiempo de control. 
208 Contenido y audiencia de los informativos: Canal Sur TV y TVE en Andalucía

\section{Variables controladas}

Los elementos de análisis inicialmente desarrollados para el control del medio prensa, se han adaptado al soporte televisivo, en cuya adaptación se han tenido en cuenta los criterios y tecnología desarrollada por el Comité de Expertos de Infoeuropa ${ }^{14}$, con el objeto de disponer de unos resultados contrastables, en sus criterios generales, al informe europeo.

Los resultados que se ofrecen, por tanto, son susceptibles de modelización matemática, cuya interpretación aporta soluciones cualitativas basadas en criterios científicos, evitando en lo posible los elementos especulativos en la interpretación de los mensajes.

Concretamente, el conjunto de variables controladas, agrupadas por bloques de información o indicadores es:

1. Datos de clasificación. Aquellos que sirven para contextualizar el contenido en el tiempo: Soportes, Banda Horaria, Día, Mes, Año, Día de la semana, y Abstracs (breve descriptor de la noticia).

2. Definición de contenidos. Variables que se utilizan para realizar el acotado temático:

2.1. Secciones. Ubican los contenidos en las secciones convencionales

y habituales de la agenda de los soportes. Dónde aparecen las noticias reflejadas en el diario: Regional, nacional, internacional, sociedad...

2.2. Temática. Ordena los argumentos en función de los contenidos a los que se refieren las noticias. De qué tipo de noticia se trata: Política, sucesos/información general, economía, deportes...

2.3. Contexto temático. Referente de las noticias: de internacional hasta local.

2.4. Categoría temática. Ubica los contenidos en categorías amplias, sintéticas, que resumen las principales casuísticas y permite controlar los niveles de importancia y/o protagonismo que reciben las distintas facetas de la vida pública y cotidiana. V.gr.: Relaciones institucionales; Vida parlamentaria; Orden público, etc.

2.5. Sujeto de la noticia. Controla las personas o instituciones a quienes se refiere la noticia, tanto los sujetos que aparecen en imágenes, como aquellos que sólo mencionan en el texto. Se consideran hasta cuatro sujetos para una misma noticia con el fin de poder estudiar las relaciones entre ellos, jerarquía otorgada, rango...

${ }^{14}$ Informe Anual FUNDESCO/APE. La Unión Europea en los medios de comunicación 1995. Madrid. Tabapress. 
Con estas variables se controla la importancia que concede cada soporte a los distintos temas en sus respectivas agendas y que, puestas en relación con otros bloques, permite obtener valoraciones cualitativas sobre diversos aspectos.

3. Autoría y origen. Controla la procedencia de las noticias, tanto en lo referido a las fuentes como al origen de la información, pudiendo discriminar la proximidad o lejanía de las fuentes y su vinculación al medio, lo que permite explorar aspectos cualitativos en relación con los sujetos, protagonismo, tratamiento, etc.

3.1. Origen informativo. Discrimina entre las distintas provincias andaluzas, otras comunidades nacionales, comunitarias,...

3.2. Fuente. Se considera un concepto de fuente más amplio que el tradicional, al incluir en esta variable las entrevistas, ruedas de prensa, etc. que proporcionan una valoración más precisa para analizar la credibilidad de la fuente como uno de los factores de persuasión.

4. Relevancia. Detecta la jerarquización que se hace de los sujetos y/o del hecho noticioso sobre la base delbase al tratamiento textual e icónico y al protagonismo que se le concede dentro de la unidad informativa -telediario.

4.1.Duración. Tiempo concedido a cada noticia, per se y con relación al tiempo total.

4.2. Tratamiento Global. Utilización de recursos audiovisuales para favorecer o aminorar el hecho o personaje: Sólo locución, locución más imágenes de archivo, más entrevistas, directo...

4.3. Presencia. Jerarquización concedida al sujeto durante su presencia en pantalla. Se controla el tratamiento que recibe en cuanto comparte, o no, noticia con otro sujeto: Presencia continua o compartida con sujetos de mayor, menor o igual rango.

Este bloque se complementa con variables clasificadas en "Otros", las cuales potencian o disminuyen los valores aquí recogidos.

5. Protagonismo informativo. Con este conjunto de variables, se busca reconocer los distintos elementos que describen la presencia informativa; en concreto, el peso específico de las instituciones y de los personajes públicos en relación con la proyección mediada de la Comunidad Andaluza. Se mide a través de la intensidad informativa y el emplazamiento que se le concede dentro del bloque.

5.1. Intensidad. Preeminencia dada al sujeto o hecho en el contexto global de la unidad informativa: avance y titulares; avance y desarrollo, etc. 
210 Contenido y audiencia de los informativos: Canal Sur TV y TVE en Andalucía

5.2. Emplazamiento en bloque. Lugar que ocupa dentro de cada bloque informativo: abre bloque, interior de bloque, etc.

6. Valoración subjetiva. Visión del analista objetivizada sobre el "valor remanente" que puede obtener de la información un supuesto espectador medio.

6.1. Valoración del contenido textual: se mide aquí el tratamiento que da el medio, a través de los presentadores, de la noticia y/o sujetos que en ella intervienen: de muy favorable a muy desfavorable.

6.2. Valoración del contenido icónico: detecta la elección de la imagen que acompaña a la noticia, pudiendo ir desde el valor muy favorable a muy desfavorable.

\subsection{Indicadores}

Con el objeto de hacer una valoración del contenido que refleje los aspectos más representativos del hecho informativo y del tratamiento que reciben las Instituciones según las distintas cadenas televisivas, se han elaborado algunos indicadores que sintetizan los conceptos-clave en un guarismo o categoría y, a la vez, establecer valoraciones comparativas entre diferentes sujetos e instituciones según soportes.

Estos indicadores son:

\section{* INDICADOR DE RELEVANCIA DE LOS SUJETOS}

Se define como la importancia que se les otorga a los sujetos en el conjunto de la unidad informativa. Para su elaboración se han ponderado las siguientes variables:

-Duración de la noticia: es el control del tiempo dedicado a cada hecho informativo. La valoración de esta variable se realiza según una categorización en segundos de las noticias, cuyos intervalos se han establecido en función de la duración global media, teniendo en cuenta los valores tipificados mediante la desviación típica del conjunto de unidades informativas.

-Tratamiento global: se trata de valorar los recursos audiovisuales utilizados para favorecer o aminorar la importancia de cada sujeto. De este modo se le otorga mayor importancia, por ejemplo, a los sujetos cuya información se cubre en directo, y menos a los que son mencionados en el transcurso de la exposición; existen valores intermedios para las distintas rutinas informativas en función de los recursos utilizados. Será por tanto distinto, a 
efectos de relevancia, que las imágenes procedan de archivo, de un reportaje o de una entrevista.

-Presencia: se define como la jerarquización concedida al sujeto cuando éste aparece en pantalla. En este sentido, se asigna un mayor valor a quienes se les otorga una presencia continua en pantalla que a los que se presentan compartiendo la imagen con otros sujetos de menor, igual o mayor rango.

El criterio que proporciona este indicador de relevancia viene dado por la siguiente relación:

\section{Indice de relevancia del sujeto $=$ Duración + Tratamiento*0,5 + Presencia}

Los resultados cuantitativos obtenidos para este indicador se agrupan en las categorías baja, media y alta, de acuerdo con los valores aportados por las combinaciones posibles entre las tres variables de la relación anterior. De este modo, aquellos sujetos que aparecen en noticias de mayor duración, con un tratamiento audiovisual más favorable y con presencia continua en pantalla, alcanzarán un grado de relevancia alta, mientras que, por ejemplo, los que forman parte de noticias de menos duración y además reciben un tratamiento menos favorable, así como una presencia compartida en la pantalla, obtendrán una categoría baja.

\section{* INDICADOR DE JERARQUÍA INFORMATIVA DE LOS SUJETOS}

Es el rango jerárquico otorgado a los sujetos; la preponderancia con que se trata a un sujeto frente a otro. La principal diferencia con respecto al indicador de relevancia es que en este nuevo índice se tienen en cuenta aspectos cualitativos del tratamiento mientras que el primero se centraba más en características cuantitativas.

Las variables analizadas en este caso son, en gran medida, la suma de las que componían los indicadores anteriores, con excepción del factor centralidad, ya que aquí no influye la procedencia del sujeto ni de la noticia.

Concretamente, el indicador de jerarquía lo componen tres factores: el de Relevancia (con las mismas variables del primer índice), el factor "Protagonismo", descrito en el indicador de vertebración, y la Valoración subjetiva, compuesto por la suma de la valoración textual e icónica. De otro modo, la jerarquía la miden la combinación de las variables: Duración, Tratamiento global, Presencia, Intensidad informativa, Emplazamiento en bloque, Valoración del contenido textual y Valoración del contenido icónico. 


\section{Contenido y audiencia de los informativos: Canal Sur TV y TVE en Andalucía}

Los tres factores se ponderan en función de que las variables que los componen sean más o menos definitorias de la jerarquía concedida a los sujetos informativos. Así, el protagonismo de la noticia está considerado como más diferenciador que la relevancia dada en el tratamiento periodístico y aún más que la valoración subjetiva del analista, dado que esta variable es la que puede introducir un mayor sesgo involuntario.

Este indicador se ha construido con los siguientes criterios operativos: Jerarquía informativa $=$ Indice de Protagonismo*0,6 + Indice de Relevancia*0,4 + Indice de Valoración subjetiva*0,2.

\section{Resultados}

El lector podrá hacerse a la idea de la cantidad de información que puede obtenerse de la puesta en relación de las variables controladas. Siquiera la distribución de frecuencias de las variables tendrían cabida en los límites de este artículo. Por ello solo nos queda la posibilidad de exponer un pequeño resumen de resultados derivados de alguno de los indicadores utilizados, como exponentes sintéticos de la riqueza de información que proporciona esta técnica de análisis ${ }^{15}$.

\section{Indicadores}

Concretamente, se van a utilizar los datos provenientes de relacionar el indicador de "Protagonismo Informativo" con las variables "Temática", "Contexto" y "Categorías informativas" anteriormente definidas, que se exponen en el cuadro de más abajo, como muestra de las posibilidades de análisis.

En las dos primeras columnas se expresa la importancia relativa de los contenidos categorizados en los criterios definidos por las variables, y en las columnas siguientes se refleja el valor alcanzado por el indicador en la categoría definida como "alta" (ver supra).

De acuerdo con lo investigado, puede afirmarse que los informativos de Canal Sur tienen un mayor componente político, así como una estructura más "abierta" (más internacional, nacional) en el reparto de los contenidos que la desconexión de TVE para Andalucía, prácticamente centrada en noticias regionales y, sobre todo, locales.

\footnotetext{
${ }^{15}$ El lector puede encontrar algunas explotaciones de esta investigación, realizadas por y con fines políticos, en los diarios El País de 2/4/98, suplemento Andalucía, pags.1 y 9 y El Correo de Andalucía, del mismo día, pg. 26.
} 
Por otro lado, respecto a la categorización temática realizada, los contenidos de Canal Sur reflejan una tendencia a la sobreinclusión de temas conflictivos/reivindicativos (30\% aproximadamente del total de sus informaciones a dedicado crisis, sucesos, conflictos institucionales) mientras TVEA dedica el $34 \%$ de su espacio informativo a actos y proyectos institucionales y solo se refiere a los temas anteriores en algo menos del $18 \%$ de las ocasiones.

También se puede comprobar cómo Canal Sur concede un mayor protagonismo -en los términos como se ha definido este concepto anteriormente- a los temas políticos de carácter autonómico y local, dentro de los que destaca cuatro clases de contenidos: relaciones institucionales, crisis/ sucesos, vida parlamentaria y presupuestos/inversiones.

La desconexión regional de TVE, por su parte, pone el acento en los temas nacionales y autonómicos de carácter general, poniendo un menor énfasis en las noticias que se refieren a crisis y relaciones institucionales.

Solo con este botón de muestra - lo que nos permite la extensión de este artículo- se pueden obtener algunas conclusiones en sentido confirmatorio de la hipótesis de partida. Parece evidente la influencia política en la construcción de la agenda informativa de los informativos de las respectivas cadenas. Mientras Canal Sur evidencia un contenido con fuerte carga dialéctica en su agenda, los informativos de TVE Andalucía parecen instalarse en contenidos más livianos, menos polémicos, casi podrían tildarse de costumbristas o "folclóricas". Cualquier andaluz mínimamente al día de la situación actual no necesitará más explicaciones heurísticas para aceptar evidencia de esta conclusión ${ }^{16}$.

Y si se acepta que los informativos de estas dos cadenas son utilizados políticamente para, por un lado, fustigar al gobierno central y, por parte de TVE-A, para rebajar el tono de la confrontación, la pregunta que surge a continuación no puede ser otra para un investigador de los efectos de la comunicación que ¿Lo consiguen?.

Obviamente, la respuesta a esta pregunta escapa de los objetivos y las posibilidades de esta investigación, pero sí se puede hacer alguna inferencia aproximativa. Veamos.

\footnotetext{
16 Por supuesto, el resto de los análisis efectuados continúan confirmando la tesis que se extrae de estos datos. Nuevamente tengo que solicitar la benevolencia del lector por no poder exponer el resto de los resultados. Igualmente, les vuelvo a remitir a los diarios El País y El Correo de Andalucía donde se publica una información "políticamente interesada" de este trabajo.
} 
214 Contenido y audiencia de los informativos: Canal Sur TV y TVE en Andalucía

\begin{tabular}{|c|c|c|c|c|}
\hline \multicolumn{5}{|c|}{$\begin{array}{l}\text { INDICADOR DE PROTAGONISMO INFORMATIVO } \\
\text { (Valores relativos de Protagonismo «Alto» en informativos de Canal Sur v.s. TVE-Andalucía) }\end{array}$} \\
\hline & $\begin{array}{l}\text { CANAL SUR } \\
(\% \text { s/Conjunto })\end{array}$ & $\begin{array}{l}\text { TVE-And. } \\
\text { (\%s/conjunto) }\end{array}$ & $\begin{array}{l}\text { Protagonismo «Alto» } \\
\text { CANAL SUR }\end{array}$ & $\begin{array}{l}\text { Protagonismo «Alto» } \\
\text { TVE-ANDALUCÍA }\end{array}$ \\
\hline \multicolumn{5}{|l|}{ TEMÁTICA } \\
\hline Política & 32,1 & 21,9 & 19,9 & 8,3 \\
\hline Inf. Gral. & 47,9 & 60,6 & 16,2 & $10,-$ \\
\hline Economía & 2,7 & 1,8 & 7,7 & - \\
\hline Deportes & 0,6 & 0,7 & 16,7 & - \\
\hline Sociedad & 12,2 & $15,-$ & 8,4 & 4,9 \\
\hline \multicolumn{5}{|l|}{ CONTEXTO } \\
\hline Internacional & 21,7 & 6,2 & 10,7 & 5,9 \\
\hline Nacional & 14,9 & 8,8 & 14,8 & 16,7 \\
\hline Autonómico & 18,3 & 20,1 & $23,-$ & 18,2 \\
\hline Provincial & 4,4 & - & 11,9 & - \\
\hline Local & 40,7 & 64,8 & 16,3 & 5,1 \\
\hline \multicolumn{5}{|l|}{ CATEGORÍAS } \\
\hline Relaciones Instituc. & 4,9 & 4,4 & 31,9 & 8,3 \\
\hline Asuntos Ordinarios & 2,7 & 2,6 & 11,5 & - \\
\hline Crisis/Sucesos & 25,7 & 14,6 & 24,6 & 12,5 \\
\hline Vida parlamentaria & 4,9 & $4,-$ & 25,5 & - \\
\hline Conflictos Instituc. & 6,5 & 2,9 & 16,2 & 12,5 \\
\hline Pptos. Inversiones & 2,2 & 0,7 & 28,6 & - \\
\hline Orden Público & 3,8 & 4,7 & 8,3 & - \\
\hline Actos Institucionales & 10,1 & 26,3 & 7,3 & 8,3 \\
\hline Proyectos Instituc. & 3,6 & 7,7 & 11,8 & - \\
\hline Otras informaciones & 35,5 & 32,1 & 9,2 & 11,4 \\
\hline
\end{tabular}




\begin{tabular}{|c|c|c|c|}
\hline \multicolumn{4}{|c|}{$\begin{array}{l}\text { PERFIL DE AUDIENCIAS DE INFORMATIVOS DE MEDIODÍA } \\
\text { (Audiencia franja horaria: 10,1\% S./Total Población) }\end{array}$} \\
\hline & $\begin{array}{l}\text { NO VEN } \\
\text { INFORMATIVOS }\end{array}$ & $\begin{array}{l}\text { VEN INFORMATIVOS } \\
\text { NACIONALES }\end{array}$ & $\begin{array}{l}\text { CANAL SUR } \\
(\text { Share } 38,8 \%)\end{array}$ \\
\hline SEXO & $\begin{array}{l}\text { Hombre } 44 \% \\
\text { Mujer } 56 \%\end{array}$ & $\begin{array}{l}\text { Hombre } 46 \% \\
\text { Mujer } 54 \%\end{array}$ & $\begin{array}{l}\text { Hombre } 48 \% \\
\text { Mujer } 52 \%\end{array}$ \\
\hline EDAD & $\begin{array}{l}15-24 \text { años } 31 \% \\
25-34 \text { años } 15 \% \\
35-44 \text { años } 11 \% \\
45-54 \text { años } 11 \% \\
\text { más } \mathbf{5 5} \text { años } \mathbf{3 1 \%}\end{array}$ & $\begin{array}{l}\text { 15-24 años } 21,3 \% \\
25-34 \text { años } 16,5 \% \\
\text { 35-44 años } 13,8 \% \\
45-54 \text { años } 12,9 \% \\
\text { más } 55 \text { años } \mathbf{3 5 , 5 \%}\end{array}$ & $\begin{array}{l}15-24 \text { años } 12,6 \% \\
\text { 25-34 años } 13,4 \% \\
\text { 35-44 años } 17,3 \% \\
45-54 \text { años } 14,2 \% \\
\text { más } \mathbf{5 5} \text { años } \mathbf{4 2 , 5 \%}\end{array}$ \\
\hline ESTUDIOS & $\begin{array}{l}\text { Est. Primarios/Sin est. 38,7\% } \\
\text { Bachiller, EGB, BUP, 55,6\% } \\
\text { Universitarios } 6,7 \%\end{array}$ & $\begin{array}{l}\text { Est. Primarios/Sin est. 45,9\% } \\
\text { Bachiller, EGB, BUP, 47.- } \% \\
\text { Universitarios 6,9\% }\end{array}$ & $\begin{array}{l}\text { Est. Primarios/Sin est. 56,6\% } \\
\text { Bachiller, EGB, BUP, 40,2\% } \\
\text { Universitarios 3,2\% }\end{array}$ \\
\hline INGRESOS & $\begin{array}{l}\text { menos de } 125 \text { mil } \mathbf{4 3 , 6 \%} \\
\text { de } 125 \text { a } 175 \text { mil } \mathbf{2 2 , 9 \%} \\
\text { de } 175 \text { a } 225 \text { mil 7,4\% } \\
\text { más } 225 \text { mil } 6 .-\%\end{array}$ & $\begin{array}{l}\text { menos de } 125 \text { mil } \mathbf{4 6 , 6} \% \\
\text { de } \mathbf{1 2 5} \text { a } \mathbf{1 7 5} \text { mil } \mathbf{2 1 , 8 \%} \\
\text { de } 175 \text { a } 225 \text { mil 7,1\% } \\
\text { más } 225 \text { mil 7,3\% }\end{array}$ & $\begin{array}{l}\text { menos de } \mathbf{1 2 5} \text { mil } \mathbf{5 9 , 4 \%} \\
\text { de } \mathbf{1 2 5} \text { a } \mathbf{1 7 5} \text { mil } \mathbf{2 7 , 9 \%} \\
\text { de } 175 \text { a } 225 \text { mil 6,3\% } \\
\text { más } 225 \text { mil 6,3\% }\end{array}$ \\
\hline OCUPACIÓN & $\begin{array}{l}\text { Trab. Cta. propia } 5.2 \% \\
\text { Trab. Cta. ajena } 18,3 \% \\
\text { Amas de casa } 25,1 \% \\
\text { Estudia } 2,3 \% \\
\text { En paro } 12,3 \% \\
\text { Jubilados } 15,8 \%\end{array}$ & $\begin{array}{l}\text { Trab. Cta. propia } 4,8 \% \\
\text { Trab. Cta. ajena 19,3\% } \\
\text { Amas de casa } 27,8 \% \\
\text { Estudia } 17,9 \% \\
\text { En paro } 10,9 \% \\
\text { Jubilados } 19,7 \%\end{array}$ & $\begin{array}{l}\text { Trab. Cta. propia } 4,8 \% \\
\text { Trab. Cta. ajena } 16,7 \% \\
\text { Amas de casa } 30,2 \% \\
\text { Estudia } 12,7 \% \\
\text { En paro } 7,9 \% \\
\text { Jubilados } 27,8 \%\end{array}$ \\
\hline $\begin{array}{l}\text { CICLO } \\
\text { DE } \\
\text { VIDA }\end{array}$ & $\begin{array}{l}\text { Soltero joven en casa paterna } \\
29,4 \% \\
\text { Adultos en casa paterna } 9,3 \% \\
\text { Solteros independientes 3,3\% } \\
\text { Parejas mayores/Adultos } \\
\text { solos } \mathbf{3 1 , 6 \%} \\
\text { Pareja joven sin hijos 4,6\% } \\
\text { Parejas con hijos<15 años } \\
11,2 \% \\
\text { Parejas con hijos de } 15,24 \\
\text { años } 10,6 \%\end{array}$ & $\begin{array}{l}\text { Soltero joven en casa paterna } \\
20,8 \% \\
\text { Adultos en casa paterna 9,3\% } \\
\text { Solteros independientes 3,7\% } \\
\text { Parejas mayores/Adultos } \\
\text { solos } \mathbf{3 6 , 3 \%} \\
\text { Pareja joven sin hijos 3,1\% } \\
\text { Parejas con hijos<15 años } \\
13,3 \% \\
\text { Parejas con hijos de } 15-24 \\
\text { años } 13,3 \%\end{array}$ & $\begin{array}{l}\text { Soltero joven en casa paterna } \\
11 \% \\
\text { Adultos en casa paterna } 7,1 \% \\
\text { Solteros independientes 1,6\% } \\
\text { Parejas mayores/Adultos } \\
\text { solos \% } \\
\text { Pareja joven sin hijos 2,4\% } \\
\text { Parejas con hijos<15 años } \\
17,3 \% \\
\text { Parejas con hijos de } 15-24 \\
\text { años \% }\end{array}$ \\
\hline HÁBITAT & $\begin{array}{l}\text { Capital } 40,6 \% \\
\text { Rural } \mathbf{5 9 , 4 \%}\end{array}$ & $\begin{array}{l}\text { Capital } 42,2 \% \\
\text { Rural } \mathbf{5 7 , 8 \%}\end{array}$ & $\begin{array}{l}\text { Capital } 38,6 \% \\
\text { Rural } \mathbf{6 1 , 4 \%}\end{array}$ \\
\hline
\end{tabular}




\section{Audiencias}

A partir de los datos incluidos en la tabla "Perfil de audiencias de informativos de mediodía", vamos a tratar de comprobar si los contenidos formalizados en los telediarios son los más adecuados para las audiencias "esperadas" por los emisores. Observando dicha tabla, se puede colegir lo siguiente:

- en todas las categorías se da un mayor sobrepeso de mujeres, pero donde más féminas se encuentran es en el informativo autonómico de televisión española y entre quienes no ven los informativos de este periodo horario; donde menos, en el target de los informativos nacionales y de Canal Sur que se adaptan más al conjunto de la población.

- los informativos de TVE-A, sobre todo, y de Canal Sur cuentan con la audiencia más madura en términos de edad. Los más jóvenes o no ven informativos o se exponen a los de las cadenas nacionales.

- los niveles de formación de las audiencias van decayendo de forma paulatina hasta llegar a TVE-A que acumula cerca de un $60 \%$ de su público entre personas con estudios primarios como máximo. Esta situación es muy parecida para Canal Sur y bastante diferente respecto a los informativos nacionales y, sobre todo, el grupo que no ve informativos. En otras palabras, se encuentra una relación inversa entre la variable formación y la audiencia de informativos regionales. Para los informativos nacionales, se mantiene una estructura de la variable formación equivalente al conjunto de la población.

- la situación económica de las audiencias muestra una situación similar a la anterior: quienes se exponen a TVE-A son los que cuentan con menor poder económico de los grupos analizados (casi tres de cada cuatro se encuentran agrupados en la categoría inferior de la variable), rasgo muy parecido entre la audiencia de Canal Sur y opuesto al de los que no ven informativos.

- los niveles de ocupación indican, nuevamente, un mayor predominio de amas de casa y jubilados, no activos, entre quienes componen la audiencia de la Televisión Española regional, después en la audiencia de Canal Sur, en los informativos nacionales -que vuelven también en esta variable a presentar unas características similares o más parecidas al conjunto de la población- $\mathrm{y}$, finalmente, donde menos se comparte este rasgo es entre las audiencias de otros programas de la banda horaria no informativos.

- la variable ciclo de vida permite comprobar cómo quienes no se exponen a los informativos de televisión son los solteros en casa paterna. 
Por su lado, el grupo más notorio en los informativos de televisión española en su desconexión regional y de Canal Sur son las parejas mayores.

- finalmente, el hábitat nos indica que en todos los casos hay un predominio de audiencia en los hábitats rurales, pero sobre todo entre las audiencias de televisión española regional y canal Sur.

Como consecuencia de todo ello se dibuja un perfil que podría resumirse de la siguiente forma: el periodo horario de los informativos cuenta con una audiencia "normal", es decir, el perfil de las audiencias de esta hora -entre las dos y las tres y media de la tarde- es homogéneo con las características del conjunto de la población. Sin embargo, entre las diversas opciones existentes, los diferentes grupos sociales "usan" los soportes conforme a las gratificaciones que reciben de ellos. Así, los más jóvenes se orientan claramente hacia las películas y series, mientras los de mayor edad se inclinan por informativos cuyos contenidos cuentan con una carga informativa menos conflictiva. En otras palabras, la audiencia de televisión entre las 14 y las 15,30 horas se corresponde con las personas que están en casa en ese momento.

La hipótesis formulada anteriormente parece cumplirse en este aspecto. Los informativos los ven las personas que se encuentran en casa y, dentro de las diferentes opciones, eligen la que más se adapta a sus gustos. Quienes se exponen a los telediarios nacionales, suelen ser personas que tienen un cierto mayor nivel formativo que la media de la población. Por su parte, los que se exponen a Canal Sur y a la desconexión de Televisión Española en Andalucía, muestran un perfil claramente de desocupado, el decir, de amas de casa e inactivos o jubilados, por lo tanto, las características personales del resto de variables aparentan un mayor sobrepeso en los rasgos que definen tradicionalmente a estas personas, es decir, menor nivel cultural, menor nivel económico, más edad y mujeres.

Nuevamente podemos hacer nuestras las afirmaciones de Aldana (1998:64-65) en el sentido de que "En buena medida el consumidor de informativos lo es porque está en casa a la hora en que los emiten (...) El consumidor de informativos es fundamentalmente un consumidor de TV y no alguien especialmente interesado por la información (...) Se consume en compañía de la familia, aunque los más jóvenes tienden a disgregarse del grupo familiar..."17

17 ALDANA, M. (1978): La información en TV: papel de los informativos en la lucha por la audiencia en A.E.D.E.M.O. $83^{\circ}$ Seminario. $14^{\circ}$ Seminario sobre audiencia de televisión. Granada. Febrero, 1998. pp. 64-65. 


\section{Conclusiones}

Los objetivos definidos para esta investigación trataban de comprobar a través de los informativos diarios como mejores exponentes de la intencionalidad ideológica, si la audiencia específica de estos programas se aparta sensiblemente de la tipología general del periodo horario y, en caso de confirmarse la hipótesis, si se podría establecer alguna relación entre el perfil de sus audiencias y los contenidos de cada informativo -supuestas gratificaciones ideológicas- o, por el contrario, si se evidencia que la audiencia de los informativos es similar a la del conjunto del periodo coloquialmente, si el público ve "lo que hay"- en cuyo caso la selección de los valores/noticia por parte de los emisores -periodistas- están en disonancia con lo que el público espera.

Los análisis efectuados tras la adecuación de las variables y la construcción de indicadores oportunos, nos permiten afirmar de una forma parcial ${ }^{18}$ las hipótesis establecidas. Efectivamente, los datos recogidos indican, por una parte, que se produce una evidente selección y tratamiento de las noticias de forma que determinadas temáticas -y sujetos- se vean realzadas, disminuidas o ignoradas. Pero también se ha podido comprobar que el público de los informativos no tiene una especial predilección por este tipo de programas; es "lo que se puede ver" y dentro de las escasas opciones disponibles se pasa de una a otra buscando "lo más distraído" (Aldana, op. cit.). Pero no es menos cierto que existe una relativa tendencia o predilección por los informativos entre las personas -más entre los varones- a partir de un cierto nivel formativo y económico ${ }^{19}$, sin que se pueda llegar a "la tan querida, por algunos, equiparación entre mi periódico=mi informativo..." (Aldana, op. cit: 65).

Por otro lado, la tendencia de los jóvenes -y de los mejor formados, también (y los jóvenes son hoy día los mejor formados)- hacia los contenidos de entretenimiento parece evidenciada en los resultados aportados por la investigación. Correlativamente, las personas mayores y las desocupadas en general se caracterizan por un mayor consumo televisivo y "la información es más importante cuando la exposición es más fuerte" (Scaglia y Souchon, 1991) ${ }^{20}$, lo que se corresponde con las relaciones encontradas.

${ }^{18}$ Cuando se trata de audiencia todas las conclusiones tienen que ser especialmente parciales.

${ }^{19}$ En mi libro Los andaluces y los medios de comunicación, (1995). Junta de Andalucía. Sevilla. pp. 137-138. se exponen los pormenores de estos fenómenos comportamentales del público.

${ }^{20}$ SCAGLIA, D. Y SOUCHON, M. (1991): "Le public de la televisión: des comportaments differents" en Les media, la publicité et la propagande.. Paris. Institut de Recherches et d'Etudes Publicitaires. 
Todo ello abunda en el corpus teórico de base por cuanto se constata una cierta selectividad de las audiencias por los contenidos que más se adaptan a sus gustos, valores y predisposiciones, dentro de las limitadas opciones disponibles. La audiencia, por lo tanto, usa la televisión como una forma de entretenimiento; el hecho de que en nuestra sociedad perduren los horarios laborales partidos proporciona dos "prime time" a los emisores 21 que aprovechan para captar al mayor público posible para, bien, ofrecerles la "información" que necesitan, o bien y además, para tratar de "enganchar" la mayor parte de audiencia y trasvasarla hacia los programas que siguen, manteniendo de esta manera los share más altos posibles en búsqueda de la rentabilidad comercial.

La multitud de implicaciones económicas, sociales, culturales, etc. que subyacen tras la situación que se acaba de apuntar sugieren nuevas hipótesis y análisis que podrían hacernos perder los objetivos concretos de este trabajo. Así pues, en lo que se refiere a la posible eficacia de la utilización de los informativos por parte de los emisores autonómicos, para transmitir a la audiencia una cierta visión de la realidad, mediante la selección y manipulación -en sentido amplio- de hechos noticiables -construcción de la agenda, podemos afirmar que solo en parte parece confirmarse, ya que:

a) el "caldo de cultivo" -léase audiencia específica- tiene sus propios criterios y opta por los espacios que más le apetecen en cada momento, con lo que es difícil inocularle la ración adecuada.

b) la tipología de las audiencias respectivas -desocupados, de baja formación, más de 50 años,...- tampoco entran en los estereotipos de líderes de opinión como para que pueda esperarse de ellos un efecto "mancha de aceite" en la difusión de los contenidos.

Así, pues, estas conclusiones podrían servir para “... recordar al planificador del peligro de esperar efectos uniformes . Diferentes personas tienden a utilizar la comunicación diferentemente bajo diferentes condiciones, lo que en definitiva, conduce a efectos diferentes" (Windahl, Signitzer y Olson, op. cit.: 219-220).

Finalmente, el objetivo instrumental de esta investigación era conseguir la puesta a punto una herramienta de análisis, sustitutiva de los tradicionales

\footnotetext{
${ }^{21}$ La existencia de dos "prime time” es inusual en los países avanzados de nuestro entorno. En España este hábito de "escuchar/ver las noticias mientras se come" está arraigado desde hace más de medio siglo, primero con la radio y, después, con la televisión "nacional” que siguió las pautas existentes.
} 
220 Contenido y audiencia de los informativos: Canal Sur TV y TVE en Andalucía

"vaciados de prensa", que responda a criterios científicos mediante la utilización de una metodología científicamente contrastable. En este sentido, creo que el objetivo ha sido perfectamente cumplido; la información que se recoge con este sistema proporciona no solo clasificaciones mucho más amplias y versátiles que los mencionados "informes de fotocopias", sino también fiables y válidas, que son los dos requisitos inexcusables que debe cumplir un instrumento de investigación para ser científicamente contrastado. 


\section{Bibliografía complementaria}

- ASA BERGER, A. (1991): Media analysis techniques.Ed.Rev.. London. Sage.

- BARDIN, Laurence (1986): Análisis de contenido. Madrid. Akal.(Edición original en francés, 1977.Presses Universitaires)

- BARWISE, P. \& EHREMBERG, A. (1992): Television and its Audience. London. Sage.

- FAGEN, Richard R. (1967): Política y Comunicación. Buenos Aires. Paidós.

- FORNER, R.M. (1992): «David contra Goliat: el triunfo de la Prensa sobre la Televisión para determinados Grupos Objetivos, en España» en Investigación y Marketing, 38, marzo, pp. 99-106.

- GRABER, D. (1987): El poder de los medios en la política. Buenos Aires. Latinoamericana. Buenos Aires.

- HALL, S. (1980): "Cultural studies: two paradigms" en Media, culture and society. A critical reader. London. Sage. Pag.33-48

- HORNIG PRIEST, S.(1966): Doing media research. An introduction. London. Sage.

- KLAPPER, J.T. (1974): Los efectos de las comunicaciones de masas. Madrid. Aguilar. (Edición original inglesa en 1960. Free Press.New York)

- KRIPPENDORFF, Klaus. (1990): Metodología de análisis de contenido. Teoría y práctica. Barcelona. (Edicción original inglesa en 1980. Sage. Newbury Park)

- McQUAIL, D. (1993): «Rethinking conceps and methods. Traditions, technologies and audiences» en M. Ferguson (ed.) Publics communications. The news imperatives. Future Directions for Media Research. London. Sage.

- MERTON, R.K. (1987): Teoría y estructura sociales. México. Fondo de Cultura Económica.

- MOORES, S. (1993): Interpreting audiences. The ethnography of media consumption. London. Sage.

- POTTER, W. James (1998): Media literacy. London. Sage.

- RASH, W. (1997): Politics on de nets. Wiring the Polítical Process. New York. W.H.Freeman and Company

- RODA, R. (1989): Medios de comunicación de masas. Su influencia en la sociedad y en la cultura contemporáneas. Madrid. CIS.

- RUIZ OLABUÉNAGA, J.I.- IZPIZUA, Ma.A. (1989): La descodificación de la Vida Cotidiana. Métodos de Investigación Cualitativa. Bilbao. Universidad de Deusto. 
222 Contenido y audiencia de los informativos: Canal Sur TV y TVE en Andalucía

- SCAGLIA, D. y SOUCHON, M. (1991): «Le public de la televisión: des comportements differents» en Les media,la publicité et la propagande. Paris. Institut de Recherches et d'Etudes Publicitaires.

- SOUCHON, M. (1993): «Defensa e ilustración de la 'audience research'» en Area 5, no 2, enero-abril, pp. 34-40.

- WEBSTER, J.G. y PHALEN, P.F. (1994): «Victim, consumer or commodity?. Audience models in communication policy» en E. Whitney, Audiencemaking: how the media create the audience. Thousand OaksLondon. Sage.

- WINDAHL, S.; SIGNITZER, B., y OLSON, J. (1992): «Using Communication Theory». London. Sage.

- WOLF, M. (1987): La investigación de la comunicación de masas. Barcelona. Paidós.

- WRIGHT, Ch. R. (1967): Comunicación de masas. Buenos Aires. Paidos.

- WRIGHT, Ch.R. (1974): Functional analysis and mass communication revisited. Blumler-Katz (eds.).1974. 